\section{Ischemic diabetic retinopathy as a possible prognostic factor for chronic kidney disease progression}

WJ Lee ${ }^{1}$, L Sobrin ${ }^{2}$, MH Kang ${ }^{1}$, M Seong ${ }^{1}$, YJ Kim ${ }^{1}$, $\mathrm{J}-\mathrm{H} \mathrm{Yi}^{3}$, JW Miller ${ }^{2}$ and $\mathrm{HY} \mathrm{Cho}^{1,2}$

\begin{abstract}
Purpose To assess the value of diabetic retinopathy (DR) severity as a possible predictive prognostic factor for the progression of chronic kidney disease (CKD). Patients and methods Retrospective cohort study. Patients (51) who were initially diagnosed with DR and CKD were enrolled and their medical records were evaluated. The following ophthalmic factors were assessed by fluorescein angiography at the initial visit: area of capillary nonperfusion, presence of neovascularization and vitreous hemorrhage, and DR grade. The effect of these factors on CKD progression over the 2-year period of the study, defined as doubling of serum creatinine or the development of end-stage renal disease requiring dialysis or renal transplant, was evaluated.

Results The study included 51 patients with DR and CKD; of these, 11 patients (21.6\%) were found to have proliferative DR (PDR) and seven patients (13.7\%) had highrisk PDR at baseline. Patients with ischemic DR, who showed extensive capillary nonperfusion ( $\geq \mathbf{1 0}$ optic disc areas) in the retina, had a greater risk for CKD progression (hazard ratio $=6.64 ; P=0.002$ ). Conclusion We found that extensive capillary nonperfusion in the retina greatly increased the risk of progression of CKD in patients with DR. This suggests that the retina and the kidney may have shared risk factors for microvascular disease secondary to diabetes mellitus, and emphasizes the need for a team approach to diabetes care.

Eye (2014) 28, 1119-1125; doi:10.1038/eye.2014.130; published online 4 July 2014
\end{abstract}

\section{Introduction}

The number of patients with end-stage renal disease (ESRD) requiring dialysis therapy is increasing worldwide. Early diagnosis and treatment of chronic kidney disease (CKD) may reduce the burden of ESRD. ${ }^{1}$ In addition, CKD is a known risk factor for cardiovascular disease, and prohibiting and delaying the progression of CKD is therefore important to prevent not only ESRD, but also cardiovascular disease., ${ }^{2,3}$

Diabetes mellitus is one of the leading causes of $\mathrm{CKD}$, and recent epidemiological studies have shown a significant rise in the prevalence of diabetes worldwide. ${ }^{4,5}$ Diabetes is a metabolic disorder of carbohydrate metabolism in which glucose is underutilized, producing hyperglycemia. The complications of diabetes include both microvascular complications (predominantly retinopathy, nephropathy, and neuropathy) and macrovascular complications. ${ }^{6}$ The complications of diabetes increase the burdens on diabetic individuals and health-care systems.

The retina and the kidney share similar microvascular complications from diabetes mellitus. These diabetic microvascular complications may have devastating consequences, including blindness and ESRD. Some authors have identified associations between the complications themselves, and one complication can serve as a risk factor for another. Recently, studies have shown that the presence of diabetic retinopathy (DR) itself may leave patients at risk for diabetic nephropathy. ${ }^{7-11}$ However, the specific prognostic factors of DR such as DR stage, presence of neovacularization (NV) or vitreous hemorrhage, and degree of nonperfusion area on fluorescein angiography
${ }^{1}$ Department of Ophthalmology, Hanyang University College of Medicine, Hanyang University Guri Hospital, Guri-City, South Korea

${ }^{2}$ Department of Ophthalmology, Harvard Medical School, Massachusetts Eye and Ear Infirmary, Uveitis and Retina Services, Boston, MA, USA

${ }^{3}$ Department of Nephrology, Hanyang University College of Medicine, Hanyang University Guri Hospital, Guri-City, South Korea

Correspondence: HY Cho, Department of Ophthalmology, Hanyang University College of Medicine, Hanyang University Guri Hospital, Guri-city, South Korea Tel: +82 31560 2353; Fax: +82 315649479 E-mail: hycho@ hanyang.ac.kr

Received: 23 November 2013

Accepted in revised form: 24 April 2014 Published online: 4 July 2014 
(FA) that predict the progression of CKD have not yet been studied.

We predicted that extensive nonperfusion area on FA, $\mathrm{NV}$, vitreous hemorrhage and higher DR stage could reflect the degree of injury in renal small vessels and predict the risk of developing ESRD. We examined whether DR increased the risk of CKD progression and examined which ocular factors predict the progression of CKD.

\section{Subjects and methods}

\section{Study design and participants}

We performed a retrospective cohort study in patients with DR and CKD to investigate whether DR is associated with the progression of CKD over a 2-year period. The medical records used in this study were collected from patients initially diagnosed with DR and CKD who visited the outpatient clinics of both the Departments of Ophthalmology and Nephrology of Hanyang University Guri Hospital between July 2007 and January 2009. This study was performed in September 2012. We only enrolled the patients who were undergoing FA for clinical purposes at their initial visit to the ophthalmology clinic. The patients were initially diagnosed with CKD by a nephrologist according to the National Kidney Foundation KDOQI (Kidney Disease Outcomes Quality Initiative) classification. ${ }^{12}$ All enrolled patients adopted prophylactic strategies such as blood pressure (BP) control, glycemic control, and dietary protein restriction in the Department of Nephrology. Exclusion criteria were history of vitrectomy, panretinal photocoagulation, intravitreal anti-vascular endothelial growth factor injection, and renal replacement therapy including dialysis and renal transplant. In addition, patients who were not regularly followed-up by nephrologists at our hospitals over the 2-year period were excluded. We also excluded patients with concomitant glomerular disease, vascular disease, tubulo-interstitial disease, or cystic disease, which are all other causes of CKD (Figure 1).

The primary outcome measure of this study was the time to the first event of serum creatinine level doubling or development of ESRD defined as dialysis or renal transplant. The primary outcome represented the progression of CKD. ${ }^{13}$

The institutional review board and ethics committee of the Hanyang University Guri Hospital approved the study, which adhered to the tenets of the Declaration of Helsinki. Patient consent was not required.

\section{Measurement of capillary nonperfusion area in fluorescein angiographic images}

FA was recorded using a high-resolution digital fundus camera (TRC-50DX; Topcon Corporation, Tokyo, Japan) when DR was initially diagnosed. A series of digital images was taken after the rapid intravenous injection of $5 \mathrm{ml} \mathrm{10 \%} \mathrm{fluorescein} \mathrm{solution.} \mathrm{FA} \mathrm{images} \mathrm{of} \mathrm{adjacent,}$ overlapping fields were cut and pasted together to form a retinal FA montage using computer-based image reconstruction software (IMAGEnet system; Topcon Corporation). With the FA montage image, we set the center as the point between the disc and the fovea. Then, an imaginary circle was drawn, the radius of which was twice the distance from the disc to the fovea. The capillary nonperfusion area was outlined manually and then the area was measured using an area measurement program (ImageJ; National Institutes of Health, Bethesda, MD, USA) within the imaginary circle. Capillary nonperfusion was defined as a dropout of the retinal capillary bed as detected in the FA image, and enhancing the contrast mode in ImageJ software emphasized the area. The disc area (DA) was measured in the same way and the nonperfusion area was expressed as compared with the DA. Two retinal specialists who were masked to any clinical information performed the measurements.

We classified the perfusion status of DR as ischemic and nonischemic based on FA characteristics. Ischemic DR demonstrated 10 or more DAs of retinal capillary nonperfusion on FA. Nonischemic DR demonstrated $<10$ DAs in the area of retinal capillary nonperfusion on FA.

\section{Estimation of glomerular filtration rate (GFR) and determination of CKD}

GFR was estimated using the Modification of Diet in Renal Disease Study equation. ${ }^{14,15}$ CKD was defined according to

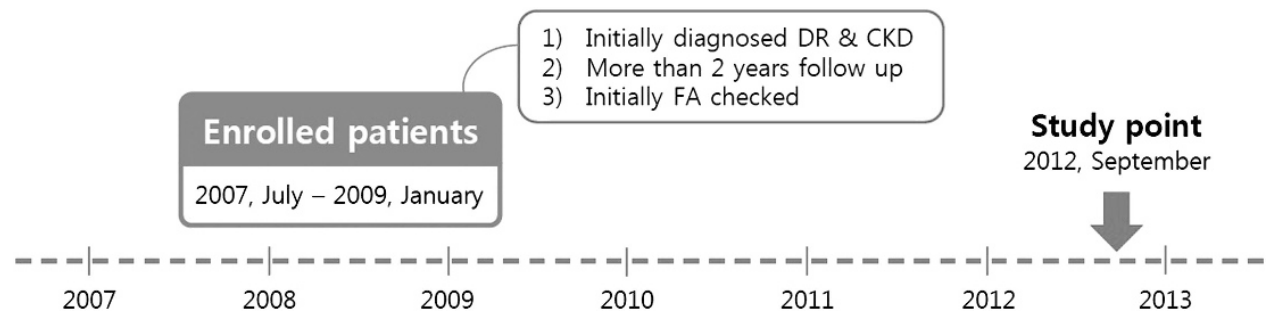

Figure 1 Flow diagram of the study subjects. CKD, chronic kidney disease; DR, diabetic retinopathy; FA, fluorecein angiography. 
the National Kidney Foundation KDOQI classification. CKD was defined as either kidney damage or $\mathrm{eGFR}<60 \mathrm{ml} / \mathrm{min} / 1.73 \mathrm{~m}^{2}$ for $\geq 3$ months. Kidney damage is defined as pathologic abnormalities or markers of damage, including abnormalities in blood or urine tests or imaging studies. ${ }^{12}$ We classified eGFR in the following ranges: stage $1, \geq 90 \mathrm{ml} / \mathrm{min} / 1.73 \mathrm{~m}^{2}$; stage $2,60-89 \mathrm{ml} / \mathrm{min} / 1.73 \mathrm{~m}^{2}$; stage $3,30-59 \mathrm{ml} / \mathrm{min} /$ $1.73 \mathrm{~m}^{2}$; stage $4,15-29 \mathrm{ml} / \mathrm{min} / 1.73 \mathrm{~m}^{2}$; and stage 5 , $<15 \mathrm{ml} / \mathrm{min} / 1.73 \mathrm{~m}^{2}$. ${ }^{12,16}$

\section{Definitions of other risk factor variables}

At the baseline examination, we checked each patient for a history of hypertension. Hypertension was diagnosed when $\mathrm{BP}$ was $>140 / 90 \mathrm{~mm} \mathrm{Hg}$ or the patient was receiving antihypertensive treatment. We also checked for the duration of hypertension, which was defined as the time from diagnosis of hypertension or the first time the patient was hospitalized. Mean data were used for analyses.

All laboratory measurements were obtained for clinical management purposes, and were not obtained at a specific time. Laboratory measurements were performed in the same laboratory and included: serum creatinine, hemoglobin, albumin, calcium, phosphate, hemoglobin A1c (HbA1c), serum lipid profile including triglyceride, low-density lipoprotein cholesterol, and high-density lipoprotein cholesterol concentration. Serum creatinine was measured by the Jaffe kinetic method. We defined 'creatinine doubling' as any increase reaching at least twice the baseline.

The presence of albuminuria was defined using urine albumin creatinine ration (UAC). UAC of $30-300 \mathrm{mg} / \mathrm{g}$ was defined as microalbuminuria and UAC over $300 \mathrm{mg} / \mathrm{g}$ was defined as overt albuminuria. ${ }^{17}$

DR was graded according to the Early Treatment for Diabetic Retinopathy Study (ETDRS) scale as nonproliferative DR (NPDR) and proliferative DR (PDR) by assessment of presence of retinal NV or abnormal growth of new retinal blood vessels in to the vitreous. High-risk PDR (HR-PDR) was defined by ETDRS guidelines. Patients who had the following risk factors were assigned to the HR-PDR group: (1) presence of neovascularization of disc (NVD) $>$ ETDRS standard photograph 10A; (2) less extensive NVD, if vitreous or pre-retinal hemorrhage was present; (3) NV of elsewhere (NVE) $\geq 1 / 2$ disc area, if vitreous or pre-retinal hemorrhage was present. ${ }^{18-21}$ For the analysis in this study, the PDR group only included those with PDR but without high-risk factors.

\section{Statistical methods}

All data are expressed as the mean \pm SD. For the analysis, we randomly selected one eye from each patient.
We categorized the patients as two groups, ischemic and nonischemic DR. Comparisons of baseline $\chi^{2}$-tests were used for categorical data and independent-sample $t$-tests were used for continuous data. Kaplan-Meier curves were used to explore differences in time to primary outcomes from the initiation of follow-up between two groups. A two-step approach was used to identify the prognostic factors associated with CKD progression. First, to identify factors associated with CKD progression, a univariate Cox regression model was used to estimate the hazard ratio and its $95 \%$ confidence interval (CI). Second, multivariate analysis using a Cox regression model was used. All the factors that were identified as affecting CKD progression by univariate analysis were included in the multivariate analysis to determine which factors were most associated with CKD progression. A forward selection method was used. $P<0.05$ was considered statistically significant in all analyses. All statistical tests were performed using the PASW Statistics 18.0 (SPSS Inc., Chicago, IL, USA).

\section{Results}

\section{Baseline characteristics}

Overall, 51 patients with DR and CKD were enrolled in this study. All of the 51 patients had type 2 diabetes. Patient distribution by the DR stage included $33(64.7 \%)$ with NPDR, 11 (21.6\%) with PDR, and 7 (13.7\%) with HR-PDR. Patient distribution by the CKD stage included $6(11.8 \%)$ in stage $1,14(27.5 \%)$ in stage $2,21(41.2 \%)$ in stage $3,7(13.7 \%)$ in stage 4 , and $3(5.9 \%)$ in stage 5 .

Patient baseline characteristics were compared between those determined to have ischemic DR and those that were nonischemic based on the extent of capillary nonperfusion on the FA montage images and are listed in Table 1 . There were 31 men and 20 women. The mean age was $58.2 \pm 12.4$ years, and mean eGFR was $55.0 \pm 29.6 \mathrm{ml} / \mathrm{min} / 1.73 \mathrm{~m}^{2}$. At baseline, 27 patients had ischemic DR and 24 patients had nonischemic DR. Albuminuria and serum creatinine levels were higher in those with ischemic DR compared with nonischemic DR, whereas eGFR, hemoglobin, and albumin values were lower.

\section{Primary outcomes}

During the 2 years of follow-up, 24 patients (47.1\%) showed CKD progression, developing one of the two primary outcomes; doubling of serum creatinine level (9 patients) or the development of ESRD (15 patients). CKD progression according to the baseline retinal perfusion status is listed in Table 2 (comparisons between the two groups). 
Table 1 Baseline characteristics of patients according to the area of capillary nonperfusion on fluorescein angiography

\begin{tabular}{|c|c|c|c|c|}
\hline Variable & Overall & $\begin{array}{c}\text { Nonperfusion }<10 D A \\
\quad \text { (nonischemic DR) }\end{array}$ & $\begin{array}{l}\text { Nonperfusion } \geq 10 D A \\
\quad \text { (ischemic DR) }\end{array}$ & $\mathrm{P}$ \\
\hline No of patients & 51 & 27 & 24 & \\
\hline Age (years) & $58.2 \pm 12.4$ & $56.7 \pm 13.9$ & $59.9 \pm 10.4$ & 0.363 \\
\hline Men $(\%)$ & 60.8 & 70.4 & 50.0 & 0.137 \\
\hline \multicolumn{5}{|l|}{ Hypertension } \\
\hline History of HTN (\%) & 80.4 & 70.4 & 91.7 & 0.056 \\
\hline Duration of HTN (years) & $5.4 \pm 4.9$ & $4.1 \pm 3.6$ & $6.6 \pm 5.6$ & 0.088 \\
\hline SBP (mm Hg) & $138.4 \pm 20.7$ & $133.6 \pm 18.7$ & $142.4 \pm 21.8$ & 0.164 \\
\hline $\mathrm{DBP}(\mathrm{mm} \mathrm{Hg})$ & $80.2 \pm 10.5$ & $81.2 \pm 10.8$ & $79.4 \pm 10.4$ & 0.582 \\
\hline \multicolumn{5}{|l|}{ Diabetes } \\
\hline Duration of diabetes (years) & $12.7 \pm 8.6$ & $10.8 \pm 7.6$ & $14.6 \pm 9.4$ & 0.126 \\
\hline $\operatorname{HbA1c}(\%)$ & $8.4 \pm 2.1$ & $8.4 \pm 1.9$ & $8.2 \pm 2.2$ & 0.752 \\
\hline Serum creatinine $(\mathrm{mg} / \mathrm{dl})$ & $1.7 \pm 1.2$ & $1.2 \pm 0.5$ & $2.2 \pm 1.5$ & 0.005 \\
\hline $\mathrm{eGFR}\left(\mathrm{ml} / \mathrm{min} / 1.73 \mathrm{~m}^{2}\right)$ & $55.0 \pm 29.6$ & $67.8 \pm 30.1$ & $40.7 \pm 21.7$ & 0.001 \\
\hline $\mathrm{UAC}(\mathrm{mg} / \mathrm{g})$ & $1803.3 \pm 2861.2$ & $555.9 \pm 1505.6$ & $3213.4 \pm 3371.7$ & 0.002 \\
\hline \multicolumn{5}{|l|}{ Albuminuria } \\
\hline None $(\%)$ & 24.5 & 46.2 & 0.0 & \\
\hline Microalbuminuria (\%) & 36.7 & 30.8 & 43.5 & 0.001 \\
\hline Overt albuminuria (\%) & 38.8 & 23.1 & 56.5 & \\
\hline BMI $\left(\mathrm{kg} / \mathrm{m}^{2}\right)$ & $24.9 \pm 2.7$ & $24.9 \pm 1.9$ & $24.9 \pm 3.3$ & 0.967 \\
\hline Triglyceride (mg/dl) & $177.9 \pm 92.9$ & $176.8 \pm 96.2$ & $179.1 \pm 91.3$ & 0.932 \\
\hline LDL cholesterol (mg/dl) & $112.9 \pm 48.2$ & $108.4 \pm 38.4$ & $117.5 \pm 57.1$ & 0.529 \\
\hline HDL cholesterol (mg/dl) & $40.1 \pm 14.2$ & $36.9 \pm 10.6$ & $43.6 \pm 16.8$ & 0.101 \\
\hline Hemoglobin $(\mathrm{g} / \mathrm{dl})$ & $11.4 \pm 2.2$ & $12.3 \pm 2.2$ & $10.5 \pm 1.8$ & 0.003 \\
\hline Albumin (g/dl) & $3.8 \pm 0.7$ & $3.9 \pm 0.6$ & $3.5 \pm 0.7$ & 0.021 \\
\hline Calcium (mg/dl) & $8.6 \pm 1.1$ & $8.6 \pm 1.4$ & $8.6 \pm 0.7$ & 0.876 \\
\hline Phosphate (mg/dl) & $3.8 \pm 1.0$ & $3.7 \pm 1.0$ & $3.9 \pm 0.9$ & 0.572 \\
\hline
\end{tabular}

Abbreviations: BMI, body mass index; DA, disc area; DBP, diastolic blood pressure; DR, diabetic retinopathy; eGFR, estimated glomerular filtration rate; FA, fluorescein angiography; HDL, high-density lipoprotein; HTN, hypertension; LDL, low-density lipoprotein; SBP, systolic blood pressure; UAC, urine albumin creatinine ratio.

Note: values are expressed as the mean \pm SD.

Table 2 Outcome according to the area of nonperfusion on fluorescein angiography

\begin{tabular}{|c|c|c|c|}
\hline Variable & $\begin{array}{c}\text { Nonischemic } D R \\
\text { nonperfusion } \\
\quad<10 D A \\
(\mathrm{n}=27)(\%)\end{array}$ & $\begin{array}{c}\text { Ischemic } D R \\
\text { nonperfusion } \\
\geq 10 \mathrm{DA} \\
(\mathrm{n}=24)(\%)\end{array}$ & $\mathrm{P}$ \\
\hline Doubling of SCr & $3(11.1)$ & $6(25.0)$ & 0.19 \\
\hline $\begin{array}{l}\text { Hemodialysis or renal } \\
\text { transplantation }\end{array}$ & $1(3.7)$ & $14(58.3)$ & $<0.00$ \\
\hline Progression of CKD & $4(14.8)$ & $20(83.3)$ & $<0.00$ \\
\hline
\end{tabular}

Abbreviations: CKD, chronic kidney; DA, disc area; DR, diabetic retinopathy; $\mathrm{SCr}$, serum creatinine.

Note: values shown are the number (percentage).

Kaplan-Meier analysis according to the perfusion status of DR is shown in Figure 2. Patients with ischemic DR had a significantly higher event rate than patients with nonischemic DR $(P<0.001)$. In our univariate model, ischemic DR, NV and vitreous hemorrhage, DR stage (PDR), albuminuria (both microalbuminuria and overt albuminuria), serum creatinine level, hemoglobin level, and albumin level were strong predictors of CKD progression (Table 3). The risk of CKD progression for patients with ischemic DR was 8.14 times that for patients with nonischemic DR (95\% CI, 2.73-24.33; $P<0.001$ ). We built a multivariate model with a stepwise forward selection process. Among the seven significant variables noted on univariate analysis, three (ischemic DR, serum creatinine level, and albumin level) had a significant independent effect on the development of outcomes in multivariate analysis.

This clarified that ischemic DR predicted the development of outcomes independently even after adjustment for other risk factors (hazard ratio, 6.64; 95\% CI, 1.96-22.52; $P=0.002)$.

\section{Discussion}

We found that patients with ischemic DR had an increased risk of CKD progression compared with 


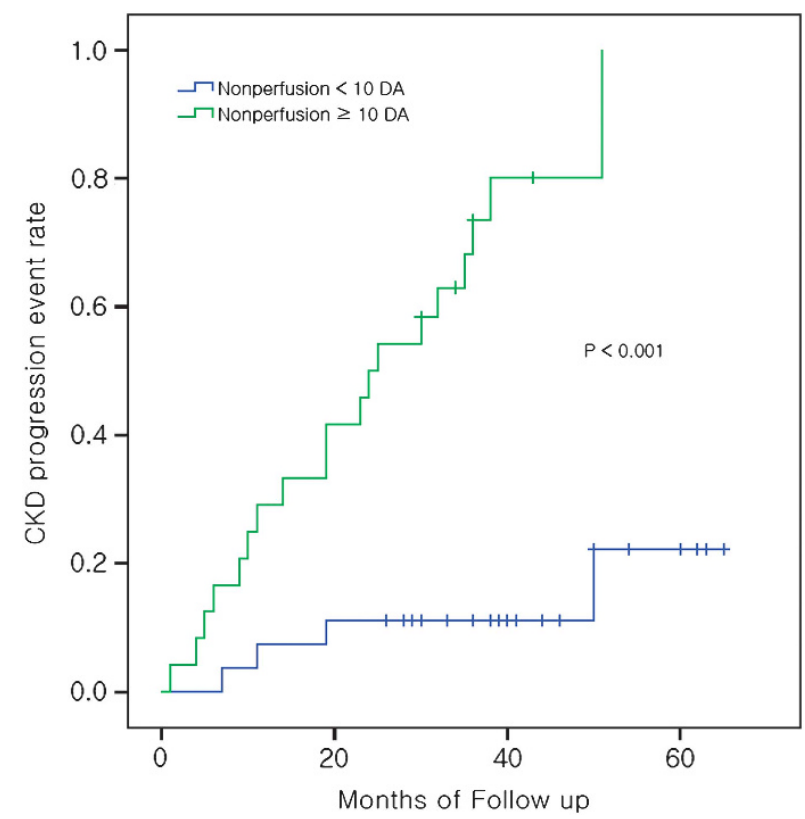

Figure 2 Kaplan-Meier estimate of event rate for primary outcomes (chronic kidney disease progression) according to the perfusion status of diabetic retinopathy on fluorecein angiography. CKD, chronic kidney disease; DA, disc area.

patients with nonischemic DR. Our findings suggest that patients with capillary nonperfusion $\geq 10$ DA on FA should be considered a high-risk population not only for DR, but also decreased kidney function; therefore, more intensive investigation of kidney function is recommended in these patients. The results of this study also support the shared microvascular damage in diabetes in the retina and the kidney.

Both the retina and the kidney are low-resistance end organs that are supplied by very small vessels. The small vessels in the retina and the kidney are highly susceptible to fluctuations in BP and blood flow. The anatomical similarities in the vascularization of the retina and the kidney give rise to complications of diabetes in the small vessels that appear in both organs. Usually, retinopathy and nephropathy progress in parallel. ${ }^{8-10}$

The patients enrolled in the current study had a high prevalence of PDR (35.3\%; including HR-PDR) and CKD over stage $3(60.8 \%)$. The data showed that patients who were coincidently diagnosed with DR and CKD had advanced microvascular complications in both the retina and the kidney.

To our knowledge, the present study is the first attempt to determine the specific prognostic factors of DR to predict the progression of CKD. We initially predicted that the extensive nonperfusion area on FA, $\mathrm{NV}$, vitreous hemorrhage, and DR staging would be possible prognostic factors for CKD progression. In the current study, we showed that the risk of reaching outcomes (CKD progression) for patients with ischemic DR was 6.64 times that for patients with nonischemic DR. It is interesting that ischemic DR was the only risk factor for CKD progression among those predicted, and that $\mathrm{NV}$ and vitreous hemorrhage were not risk factors for CKD progression. NV, vitreous hemorrhage, or DR staging are important indicators of DR severity, but have less predictive value than retinal ischemia for CKD progression. This might be owing in part to the DR severity of the study population. We only enrolled the patients who were examined with FA and this may have selected a more severe population. A follow-up study should examine a population with a greater distribution of DR severity.

We additionally performed a correlation analysis of continuous DA of the nonperfusion area with changes in serum creatinine. The nonperfusion area of initial FA exam was significantly correlated with the ratio of last serum creatinine to initial creatinine level (Spearman correlation; $\rho=0.524, P<0.001$ ) (Supplementary File). Although this additional analysis was simplified, the result could strengthen the hypothesis that ischemic DR could be a possible prognostic factor for CKD progression.

The detection of retinopathy is easy via regular fundus examination (or fundus photography), but the diagnosis of early-stage nephropathy requires microalbuminuria to be determined via urine analysis. ${ }^{22}$ Fundus examinations can detect retinal vascular changes noninvasively and directly, and the eye is the only organ in the body that allows direct visualization of microvascular changes. In regular examinations of patients with DR, FA (with minimal invasiveness) can also supplement the clinical assessment of DR, demonstrating microaneurysms, capillary nonperfusion, intraretinal microvascular abnormality, and NV. Our study showed that ischemic $\mathrm{DR}$ is valuable to predict the progression of $\mathrm{CKD}$, which means that FA for periodic DR examination can be useful not only in detecting the progression of DR but also in predicting the progression of CKD.

We categorized the patients in two groups, ischemic and nonischemic DR. We set 10 DA of nonperfusion as the reference point because 10 DA has been widely used in the evaluation and treatment of central retinal vein occlusion. $^{23}$

Our study has several limitations. First, because of the retrospective nature of this study, we could not account for all possible confounding factors that could affect CKD progression, such as smoking and family history. Second, there were some limitations in image processing. Images in the periphery were misaligned on the FA montage, and because we placed the three-dimensional retina on a two-dimensional canvas, individual FA images in the periphery were stretched and distorted. ${ }^{24}$ Although we 
Table 3 Associations of risk of primary outcomes (chronic kidney disease progression) with baseline factors

\begin{tabular}{|c|c|c|c|c|}
\hline \multirow[t]{2}{*}{ Variable } & \multicolumn{2}{|l|}{ Univariate } & \multicolumn{2}{|l|}{ Multivariate } \\
\hline & Hazard ratio $(95 \% \mathrm{CI})$ & $\mathrm{P}$ & Hazard ratio $(95 \% \mathrm{CI})$ & $\mathrm{P}$ \\
\hline Nonperfusion area $\geq 10 \mathrm{DA}$ & $8.14(2.73-24.33)$ & $<0.001$ & $6.64(1.96-22.52)$ & 0.002 \\
\hline Neovascularization and vitreous hemorrhage on FA & $3.01(1.26-7.19)$ & 0.013 & $0.91(0.29-2.78)$ & 0.871 \\
\hline \multicolumn{5}{|l|}{$D R$ stage $($ Ref $=N P D R)$} \\
\hline PDR & $3.51(1.28-9.58)$ & 0.014 & $0.75(0.17-3.36)$ & 0.706 \\
\hline HR-PDR & $2.50(0.82-7.61)$ & 0.107 & $1.03(0.29-3.65)$ & 0.961 \\
\hline Age (years) & $1.00(0.97-1.04)$ & 0.957 & & \\
\hline Men & $0.62(0.27-1.44)$ & 0.268 & & \\
\hline History of HTN & $1.85(0.54-6.30)$ & 0.326 & & \\
\hline Duration of HTN (years) & $1.07(0.97-1.18)$ & 0.198 & & \\
\hline $\mathrm{SBP}(\mathrm{mm} \mathrm{Hg})$ & $1.00(0.98-1.02)$ & 0.984 & & \\
\hline $\mathrm{DBP}(\mathrm{mm} \mathrm{Hg})$ & $1.00(0.97-1.03)$ & 0.999 & & \\
\hline Duration of diabetes (years) & $0.99(0.95-1.04)$ & 0.725 & & \\
\hline $\operatorname{HbA1c}(\mathrm{g} / \mathrm{dl})$ & $0.94(0.74-1.19)$ & 0.589 & & \\
\hline \multicolumn{5}{|l|}{ Albuminuria $($ Ref $=$ None $)$} \\
\hline Microalbuminuria & $9.91(1.26-77.83)$ & 0.029 & $1.04(0.08-13.97)$ & 0.978 \\
\hline Overt albuminuria & $17.07(2.13-137.04)$ & 0.008 & $0.99(0.06-16.91)$ & 0.997 \\
\hline Serum creatinine $(\mathrm{mg} / \mathrm{dl})$ & $1.63(1.22-2.18)$ & 0.001 & $1.37(1.01-1.86)$ & 0.044 \\
\hline BMI $\left(\mathrm{kg} / \mathrm{m}^{2}\right)$ & $1.06(0.88-1.28)$ & 0.545 & & \\
\hline Triglyceride (mg/dl) & $1.00(0.99-1.00)$ & 0.827 & & \\
\hline LDL cholesterol (mg/dl) & $1.01(0.99-1.01)$ & 0.173 & & \\
\hline HDL cholesterol (mg/dl) & $1.02(0.99-1.06)$ & 0.250 & & \\
\hline Hemoglobin $(\mathrm{g} / \mathrm{dl})$ & $0.73(0.56-0.94)$ & 0.015 & $1.03(0.73-1.45)$ & 0.879 \\
\hline Albumin $(\mathrm{g} / \mathrm{dl})$ & $0.49(0.29-0.84)$ & 0.009 & $0.46(0.24-0.87)$ & 0.017 \\
\hline Calcium (mg/dl) & $0.86(0.60-1.24)$ & 0.419 & & \\
\hline Phosphate (mg/dl) & $1.21(0.79-1.84)$ & 0.390 & & \\
\hline
\end{tabular}

Abbreviations: BMI, body mass index; CI, confidence interval; DA, disc area; DBP, diastolic blood pressure; DR, diabetic retinopathy; FA, fluorescein angiography; HDL, high-density lipoprotein; HR-PDR, high-risk PDR; HTN, hypertension; LDL, low-density lipoprotein; NPDR, nonproliferative DR; PDR, proliferative DR; SBP, systolic blood pressure.

Cox regression model.

Multivariate: forward (conditional).

limited the individual fixed imaginary circle on the FA montage, anatomical variation may have caused the evaluated area to vary. We measured the nonperfusion area relative to the individual disc area, but normal disc size can also vary. In addition, the nonperfusion area under vitreous hemorrhage could not be evaluated owing to blockage of fluorescence. Third, all of the patients enrolled in the current study were tested with FA. In common practice, FA is not routinely performed. This means that the patients in the current study had relatively advanced DR status at the initial visit and this may have caused a selection bias. Fourth, serum creatinine can fluctuate extensively, especially in diabetes, and so the definition of baseline and doubling of creatinine levels may also vary. The average level of creatinine over a time period might be a preferred measure and this should be considered in a future study.

Ischemic DR characterized by extensive capillary nonperfusion ( $\geq 10$ DA) on FA appears to predict the risk of CKD progression in patients with DR and CKD. This suggests that the retina and kidney share risk factors for microvascular disease secondary to diabetes mellitus, and emphasizes the need for a team approach to diabetes care. When ophthalmologists find ischemic DR on FA during regular DR examination, timely and prompt referral to a nephrologist is recommended.

\section{Summary}

What was known before

- The relationship between diabetic retinopathy (DR) and diabetic nephropathy $(\mathrm{DN})$ has been reported, and the prescence of DR itself may reveal patients at risk of DN.

What this study adds

- Ischemic DR characterized with extensive capillary nonperfusion is a possible prognostic factor for the progression of chronic kidney disease.

\section{Conflict of interest}

The authors declare no conflict of interest. 


\section{Author contributions}

HYC had full access to the data in the study and takes responsibility for the integrity of the data and the accuracy of the data analysis.

\section{References}

1 Foley RN, Collins AJ. End-stage renal disease in the United States: an update from the United States Renal Data System. J Am Soc Nephrol 2007; 18: 2644-2648.

2 Go AS, Chertow GM, Fan D, McCulloch CE, Hsu CY. Chronic kidney disease and the risks of death, cardiovascular events, and hospitalization. $N$ Engl J Med 2004; 351: 1296-1305.

3 Schiffrin EL, Lipman ML, Mann JF. Chronic kidney disease: effects on the cardiovascular system. Circulation 2007; 116: 85-97.

4 Keane WF, Brenner BM, de Zeeuw D, Grunfeld JP, McGill J, Mitch WE et al. The risk of developing end-stage renal disease in patients with type 2 diabetes and nephropathy: the RENAAL study. Kidney Int 2003; 63: 1499-1507.

5 Wild S, Roglic G, Green A, Sicree R, King H. Global prevalence of diabetes: estimates for the year 2000 and projections for 2030. Diabetes Care 2004; 27: 1047-1053.

6 Girach A, Vignati L. Diabetic microvascular complications-can the presence of one predict the development of another? J Diabetes Complications 2006; 20: 228-237

7 El-Asrar AM, Al-Rubeaan KA, Al-Amro SA, Moharram OA, Kangave D. Retinopathy as a predictor of other diabetic complications. Int Ophthalmol 2001; 24: 1-11.

8 Karlberg C, Falk C, Green A, Sjølie AK, Grauslund J. Proliferative retinopathy predicts nephropathy: a 25-year follow-up study of type 1 diabetic patients. Acta Diabetologica 2012; 49: 263-268.

9 Pedro RA, Ramon SA, Marc BB, Juan FB, Isabel MM. Prevalence and relationship between diabetic retinopathy and nephropathy, and its risk factors in the North-East of Spain, a population-based study. Ophthalmic Epidemiol 2010; 17: 251-265.

10 Romero P, Salvat M, Fernández J, Baget M, Martinez I. Renal and retinal microangiopathy after 15 years of follow-up study in a sample of type 1 diabetes mellitus patients. J Diabetes Complications 2007; 21: 93-100.

11 Grunwald JE, Alexander J, Ying GS, Maguire M, Daniel E, Whittock-Martin R et al. Retinopathy and chronic kidney disease in the Chronic Renal Insufficiency Cohort (CRIC) study. Arch Ophthalmol 2012; 130(9): 1136-1144.

12 Foundation NK. K/DOQI clinical practice guidelines for chronic kidney disease: evaluation, classification, and stratification. Am J Kidney Dis 2002; 39: S1-266.

13 Kobayashi M, Hirawa N, Morita S, Yatsu K, Kobayashi Y, Yamamoto $Y$ et al. Silent brain infarction and rapid decline of kidney function in patients with CKD: a prospective cohort study. Am J Kidney Dis 2010; 56: 468-476.

14 Matsuo S, Imai E, Horio M, Yasuda Y, Tomita K, Nitta K et al. Revised equations for estimated GFR from serum creatinine in Japan. Am J Kidney Dis 2009; 53: 982-992.

15 Vervoort G, Willems HL, Wetzels JF. Assessment of glomerular filtration rate in healthy subjects and normoalbuminuric diabetic patients: validity of a new (MDRD) prediction equation. Nephrol Dial Transplant 2002; 17: 1909-1913.

16 Patel SS, Kimmel PL, Singh A. New clinical practice guidelines for chronic kidney disease: a framework for K/DOQI. Semin Nephrol 2002; 22: 449-458.

17 American Diabetes Association. Report of the expert committee on the diagnosis and classification of diabetes mellitus. Diabetes Care. 1997; 20: 1183-1201.

18 Diabetic Retinopathy Study Coordinating Center. Diabetic Retinopathy Study: mannual of operations. Diabetic Retinopathy Study Coordinating Center: Baltimore, 1972.

19 Early Treatment Diabetic Retinopathy Study Research Group. Grading diabetic retinopathy from stereoscopic color fundus photographs: an extension of the modified Airlie House classification. ETDRS report number 10. Ophthalmology 1991; 98(5 Suppl): 786-806.

20 Klein R, Klein BE, Moss SE, Cruickshanks KJ. The Wisconsin Epidemiologic Study Of Diabetic Retinopathy. XIV. Ten-year incidence and progression of diabetic retinopathy. Arch Ophthalmol 1994; 112: 1217-1228.

21 Klein R, Klein BE, Moss SE, Cruickshanks KJ. The Wisconsin Epidemiologic Study of Diabetic Retinopathy: XVII. The 14-year incidence and progression of diabetic retinopathy and associated risk factors in type 1 diabetes. Ophthalmology 1998; 105: 1801-1815.

22 Snyder S, Pendergraph B. Detection and evaluation of chronic kidney disease. Am Fam Physician 2005; 72: 1723-1732.

23 Baseline and early natural history report. The Central Vein Occlusion Study. Arch Ophthalmol 1993; 111: 1087-1095.

24 Mahurkar AA, Vivino MA, Trus BL, Kuehl EM, Datiles 3rd MB, Kaiser-Kupfer MI. Constructing retinal fundus photomontages. a new computer-based method. Invest Ophthalmol Vis Sci 1996; 37: 1675-1683.

Supplementary Information accompanies this paper on Eye website (http://www.nature.com/eye) 